\title{
BMJ Open Wraparound care for youth injured by violence: study protocol for a pilot randomised control trial
}

\author{
Carolyn Snider, ${ }^{1,2}$ Depeng Jiang, ${ }^{3}$ Sarvesh Logsetty, ${ }^{4}$ Trevor Strome, ${ }^{1}$ \\ Terry Klassen ${ }^{2}$
}

To cite: Snider C, Jiang D, Logsetty S, et al. Wraparound care for youth injured by violence: study protocol for a pilot randomised control trial. BMJ Open 2015;5:e008088. doi:10.1136/bmjopen-2015008088

- Prepublication history for this paper is available online. To view these files please visit the journal online (http://dx.doi.org/10.1136/ bmjopen-2015-008088).

Received 3 March 2015 Accepted 14 April 2015

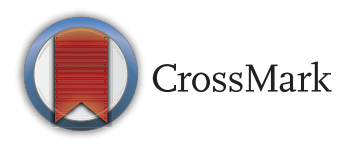

For numbered affiliations see end of article.

\section{Correspondence to} Dr Carolyn Snider; csnider@chrim.ca

\section{ABSTRACT}

Introduction: Injury by violence is the fourth cause of death and the leading reason for a youth to visit an emergency department (ED) in Canada. In Winnipeg, $20 \%$ of youth who visit an ED with an injury due to violence have a second visit for a subsequent violent injury within 1 year. Youth injured by violence are in a reflective and receptive state of mind, rendering the ED setting appropriate for intervention.

Methods and analysis: This protocol describes a wraparound care model delivered by a support worker with lived experience with violence, supported by social workers and links to multiple community partners. Support workers will be on call $24 \mathrm{~h}$ a day, 7 days a week in order to start the intervention in the ED and take advantage of the 'teachable moment'. The protocol is of a pilot randomised control trial to assess the feasibility of a randomised control trial designed to assess efficacy. For the pilot trial, we will assess recruitment, treatment fidelity, participant adherence and safety. The intervention arm will receive wraparound care initiated at the time of their visit for injury due to violence. The control arm will receive standard care. We will use an adapted preconsent randomisation methodology. This intervention has been developed using an integrated knowledge translation approach.

Discussion: Interventions delivered in the ED for youth injured by violence require an approach that is appropriate for the unique situation the youth are in. Ethics: The University of Manitoba Health Research Ethics Board (HS 16445 (Cohort study) and HS 16444 (WrapAround Care study) granted ethical approval.

Trial registration number: NCT01895738.

\section{INTRODUCTION}

\section{Background and rationale}

Youth injured by violence is a major concern in Canada. Youth homicide is the fourth most common cause of death among individuals aged 15-24 years in Canada. ${ }^{2}$ For every youth homicide, there are 115 emergency department (ED) visits and 12 admissions to hospital for intentional injury. ${ }^{3}$

\section{Strengths and limitations of this study}

- Application of a rigorous randomised controlled trial design to study a behavioural intervention with a difficult to follow population.

- Ethical concerns with the vulnerability of youth participants are addressed in this design in a unique way, yet maintain the rigour required for a randomised controlled trial.

- Demonstrates the importance of involving key stakeholders in the development of a research project.

This is not a multicentre trial.

In Manitoba, the province with one of the highest homicide rates in Canada, this issue is of special concern. ${ }^{4}$ Over the past 10 years in Canada, $26 \%$ of homicide victims and $44 \%$ of those accused were youth. ${ }^{5}$ Even more concerning is the extremely high repeat injury rate associated with violence. Injury is a chronic, recurring disease. Sims et $a l^{6}$ first introduced this concept in 1989 when they demonstrated that urban trauma had a recurrence rate of $44 \%$ and a 5 -year mortality rate of $20 \%$. In Winnipeg, $20 \%$ of youth injured by violence visited the same ED in the next year with a repeat injury due to violence. ${ }^{7}$ Currently, the standard of care for this vulnerable population is to discharge them from our EDs with no effort to prevent the next injury.

Canada's Aboriginal population is disproportionally affected by violent injury. A study of injury rates in Ontario First Nation Communities demonstrated an incidence of intentional injury $2.7 \times$ higher among the First Nations population than the general Ontario population. ${ }^{8}$ It is essential to acknowledge the historical trauma and intergenerational grief that permeates through our Aboriginal community and its role in leading youth to violent injury. Many had parents and grandparents who attended residential schools, experiencing abuse and an 
absence of parenting. This has led to homes impacted by addictions, family disintegration, childhood neglect and abuse. Children are growing up in and out of the child welfare system with a lack of consistent role modelling resulting in increased involvement in gangs. Gang-associated youth acknowledge that they joined gangs for protection, and for support and a sense of belonging that they felt were missing in their own homes.

While race information is not universally collected in EDs in Canada, it is acknowledged that a majority of the youth injured in Winnipeg are Aboriginal. It is clear that we must support these youth with Aboriginal-informed care and access to community supports within their own culture.

\section{Opportunities for secondary prevention}

Hospital-based interventions using the 'teachable moment' are ideal for use by clinicians, as they are applied to an individual who has had a significant health event thus potentially making the person more responsive to change. Johnson et at showed that youth injured by violence are in a reflective and receptive state of mind, rendering the hospital setting appropriate for intervention. Many postulate that the hospital setting can be used to create a link between the injured, and mentors who can engage with the youth and family in making changes in order to prevent future violence.

\section{Secondary prevention development}

This proposal was developed using a community-based participatory research approach. The team includes clinicians, researchers, community youth workers with expertise in wraparound care, Aboriginal elders and former gang members. Wraparound care is a model of care that puts the youth at the centre of the care model. Youth work with a support worker to determine the issues that they feel put them at risk and then build goals on trying to address those issues. The youth and support worker develop a team of individuals and organisations that help them reach that goal. This team may include family members and/or professionals whom the youth trusts. ${ }^{10}$

The first key to this intervention is the establishment of a relationship between support worker and youth at the time of injury - that is, during the teachable moment. The second key is that the support worker has extensive 'lived experience'. Many youth affected by violence have been marginalised by society and have significant trust issues with figures of traditional power (ie, doctors, social workers, etc). It is imperative that the support worker establishes a trusting relationship quickly and acts as a role model. In the 'Ceasefire Chicago' violence intervention programme, support workers with lived experience (ie, those who were former gang members or who had served time for violent crimes) were more likely to effectively influence high-risk individuals to avoid resorting to violence. ${ }^{11}$ Finally, in our community-based consultations with youth affected by violence, it was clearly communicated that the support workers be people with whom they could identify. ${ }^{12}$ The third key is that the care programme is individualised for and by each youth. The support worker enables the youth to make goals and provides the necessary steps to achieve them-for example, showing them how to find education programmes (not applying on their behalf). This is important in building the personal agency necessary to successfully reach larger goals. The final key is the opportunity to access community supports within their own culture. All of our Aboriginal former gang-member coapplicants felt strongly that developing a sense of belonging and identity within their Aboriginal culture was essential to their transition from gang life. This need has been echoed by collaborators within the Newcomer youth community.

\section{Need for a trial}

While many believe that interventions using support workers with lived experience who approach the patient in the ED are key to reducing injury, evidence does not yet exist to support their efficacy in this outcome. Youth who choose to exit gangs can be at risk of retaliation. If our intervention does not keep them safe, we must acknowledge that this may not be the most appropriate solution. Given the cost of this intervention and the potential for harm, significant equipoise exists. To date, no study has been completed using a rigorous randomised controlled trial (RCT) to evaluate the efficacy of hospital-based violence interventions on reducing repeat injury among youth. Our proposal is designed to support this needed evaluation.

\section{Study objectives}

The objective of our study is to determine the feasibility of a large RCT that will test the efficacy of connecting an injured youth at the time of injury with a support worker in reducing the incidence of subsequent violent injury versus standard of care. The support worker will provide wraparound care to address the factors that have placed the individual at risk for violence. Success of the pilot study will include achieving goals related to recruitment, treatment fidelity and participant adherence. Additionally, safety of the participants will be measured. We will ensure all the outcomes of the main RCT are indeed measureable. Finally, a pilot RCT will ensure improved clinical experience of the full-intervention team prior to the main trial. Success of the pilot study will result in an application for a full-efficacy trial.

\section{METHODS}

\section{Design}

Our study team raised concerns of causing emotional trauma to this vulnerable population through a traditional randomisation design. As such, in order to ensure autonomy over their information while maintaining an RCT design, we will use an adapted preconsent 
randomisation methodology (an adapted Zelen's design) that is also consistent with the cohort multiple RCT design. ${ }^{13} 14$

All eligible patients for the cohort will be asked by a research assistant to participate in an observational study that follows their administrative data, available through Manitoba Centre for Health Policy (MCHP), and to perform chart reviews on their visits to hospitals in Winnipeg as well as to give permission to contact them in the future to participate in potential interventions. Any individual entered into the cohort who agrees to be randomised for potential interventions will then immediately be entered into the central randomisation website (randomize.net) using an iPad in the ED. Randomize.net is a comprehensive and secure online centralised randomisation service that uses random sequence generation and provides random varying block sizes. If individuals are randomised to treatment, they would then be told about the treatment by the support worker and asked to consent to the treatment arm; those randomised to the control will not require further consent but will be contacted at the end of 1 year (after their data collection is complete); thus creating a waitlist control. Consent to further treatment would be sought at that time (figure 1).

\section{Study setting}

In Winnipeg, all traumas are directed to Winnipeg Health Sciences Centre (HSC). It has two EDs-the

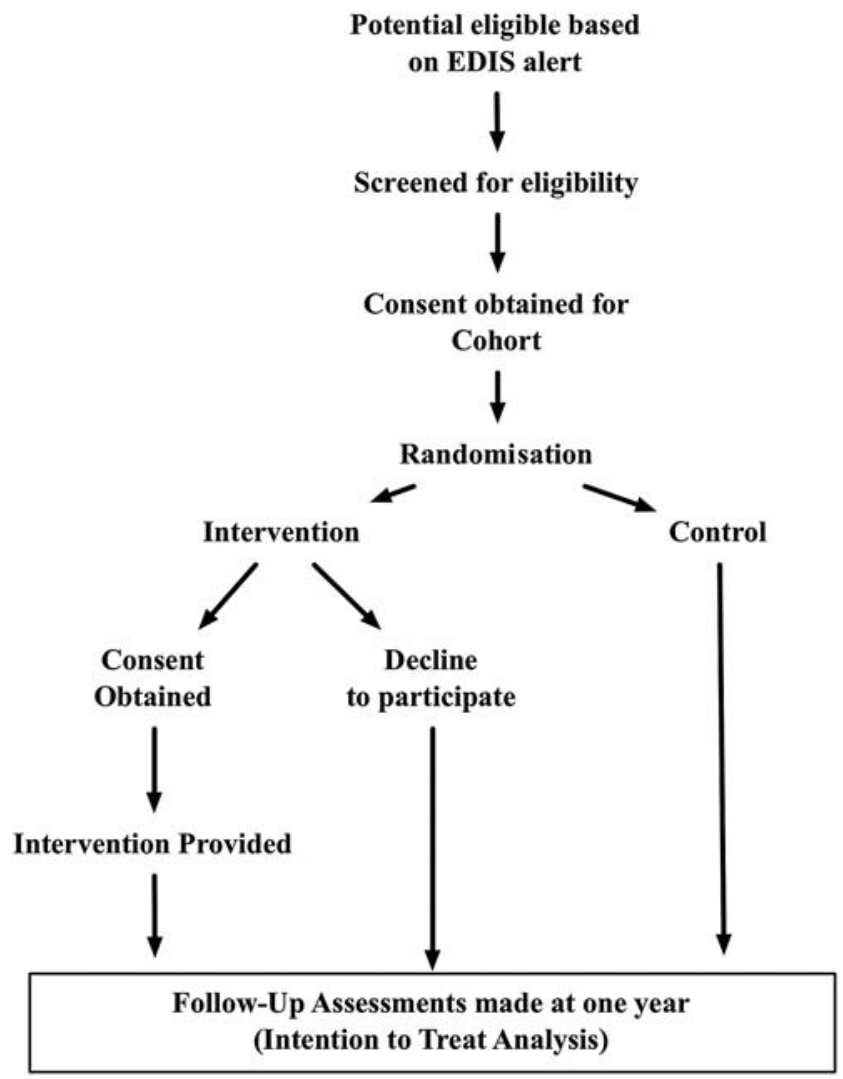

Figure 1 Study flow diagram (EDIS, emergency department information system). adult and children's sites. This collection of hospitals is located centrally in the most at-risk area of the city. The majority of violent injuries are seen at these sites. Our first site, the adult $\mathrm{ED}$, has over 60000 annual $\mathrm{ED}$ visits. The HSC trauma service sees 350 major traumas and about 2000 minor traumas per year. Our second site, the children's ED, has over 50000 annual visits to their ED, that sees the majority of children under 17 (except those with penetrating injury). These two EDs are separated by a short hallway and share an ambulance bay; however, they are considered two distinct sites with separate staff.

\section{Inclusion criteria}

- Aged 14-24 years.

- Presentation to the ED with an injury caused by interpersonal violence. (Injury is defined as an injury such as a gunshot wound, stab wound, injury due to blunt object or injury due to bodily force or intentional overdose).

\section{Exclusion criteria}

- Unable to consent due to language or pre-existing severe cognitive deficits.

- Transfer from hospital $>1 \mathrm{~h}$ from Winnipeg.

- Presentation due to intimate partner violence, sexual assault, child maltreatment or self-harm.

\section{Intervention arm}

Day 1: Youth randomised to wraparound care will be met by the support worker at the bedside in the ED or on the trauma ward as soon as the youth is awake and clear enough to complete the appropriate consents/assents. The support worker will work to establish a trusting relationship with and provide immediate support to the participant.

Day 2: The support worker will meet with the participant in the community (or in hospital if admitted) to continue to develop their relationship, review the wraparound care programme and to start enquiring about issues that the youth may wish to address during their participation. The youth will be provided with a simple activated mobile phone so that they can keep in touch with their support worker. This phone will also be used for follow-up surveys (using the fully automated MOSIO SMS survey system) to measure adherence and three secondary outcomes.

Week 1: In week 1, the support worker will attempt to be in contact with the youth every day either by phone, text or in person. At the end of week 1, the first team meeting with the participant, their support worker, social worker, addictions and mental health counsellor and family counsellor (and potentially family members if the youth wishes) will occur. During this meeting, the youth will identify three main areas of risk that they would like to improve on. The team will form strategies with the youth on ways to meet these goals. Youth will be encouraged to have at least one goal to be engaged in 
productive activity by the end of 1 year (ie, school, job training, employment or volunteer work).

Weeks 2-4: The support worker will meet the participant at least twice in person and at least confer once by phone or text.

Months 2-12: The support worker will check in at least once per week in person with their participants, and phone and text as required (minimum $2 \times$ per week), unless decided and documented at a team meeting (which includes the participant) for an alternative schedule (ie, a youth doing very well). In addition to main goals, youth will be asked to set weekly goals and support workers will help ensure that these are realistic, and then follow-up with them on this goal during a weekly check-in. There will be monthly team meetings with each youth to discuss their main goals.

\section{Control arm}

Those randomised to the control arm will be offered standard of care. ED doctor of medicines, registered nurses and social workers have access to a list of community contacts that they can use; however, provision of this list to the youth will depend entirely on the ED clinician's actions and should truly represent standard of care. The control group will then be contacted at the end of 1 year to offer them the intervention (waitlist control) after their trial data collection is completed.

\section{Sample size}

The proposed sample size for the pilot study is based on being able to recruit enough patients to feasibly perform the larger RCT. Thus, this recruitment goal of 180 (90 to each arm) is based on the sample size calculation of the larger trial. The sample size for the main RCT was calculated using a $20 \%$ baseline rate of repeat injury due to violence (based on a chart review of 4 months of visits to the sites) and an effect size of a $40 \%$ relative decrease (absolute $8 \%$ decrease) in repeat injury due to violence in the intervention arm. The effective size of an absolute $8 \%$ (40\% relative) decrease in a repeat intentional injury was determined using consultations with clinicians, community advisors (which included youth with lived experience, community youth workers and directors of local violence intervention programmes) and colleagues who run similar programmes in the USA (through the National Network of Hospital-based Violence Intervention Programmes research committee).

Randomised at a 1:1 ratio, the sample size required is 330 per arm (660 total) with a power of $80 \%$ and a twotailed significance level of 5\%. In Zelen's prerandomisation consent, one must include a percent expected to not consent postrandomisation in order to determine the overall number to be randomised. ${ }^{15}$ We have estimated this to be $10 \%$ based on our proof-of-concept work carried out prior to this pilot trial and will need to randomise 726 youth in order to reach 330 in each arm. As our primary outcome is measured using administrative data and chart review, which are included in the overall consent, theoretically we should be able to determine the outcome in $100 \%$ of subjects, even if the youth are lost to follow-up.

\section{Outcomes of pilot RCT}

\section{To determine feasibility of recruitment for main RCT}

We estimate that our sample size for the main trial must be 330 per arm (660 total). In order to ensure sufficient recruitment over 44 months plus 12-month follow-up for each subject and analysis time within a 5-year time frame, we estimate that we will need to randomise 180 youth to our pilot study in 1 year (ie, 90 to each group). Objectives set to achieve this recruitment target include: $70 \%$ of the potentially eligible (based on automatic notifications from an ED information system) are screened, consent obtained in $>32 \%$ of eligible patients for observational trial, randomise $>98 \%$ of those consenting in observation trial and $>90 \%$ of those randomised to the intervention consent. (We expect this high consent rate based on the preconsent process and our proof-of concept experience).

\section{To assess fidelity to treatment protocol}

We have set the treatment protocol based on experience from our proof-of-concept work and our community partners. In behavioural interventions, fidelity measures are considered the quality control measure of ensuring the intervention is being implemented as intended. ${ }^{16}$ Fidelity preserves internal validity against type I and II errors, it improves power by decreasing the variability in treatment effect and allows replication, dissemination and implementation in practice. Successful fidelity will be defined as $>85 \%$ of contacts (ie, check-ins, phone calls, team meetings) attempted by the support worker.

\section{To assess participant adherence}

Participant adherence refers to both the receipt of the intervention (the development of a strong and effective alliance between the case manager and youth) and enacting on the intervention (accessing resources). In behavioural intervention studies it is important to demonstrate that the actual intervention is occurring as designed. If we do not achieve adherence to the intervention, then our comparison of outcomes does not truly measure the difference between intervention and control. Successful adherence will be assessed using the Working Alliance Inventory-Short Form (WAI-S), which measures the bond between the support worker and participant, and the agreement on tasks and goals set. ${ }^{17}$

\section{To assess serious adverse events}

Given that youth injured by violence, especially those who are gang-involved, are at high risk of retaliatory violence, we must ensure participants do not experience increased harm as part of the intervention. Programme staff will fill in an incident report form for all adverse events. Programme staff will discuss adverse events at 
each weekly meeting, and workers and counsellors will be required to fill in an incident report form should an incident occur. These will be reviewed immediately with the principal investigator and, if necessary, the Data and Safety Monitoring Board (DSMB). At the end of 1 year of follow-up of the first 180 participants, the DSMB will be presented with data to assess for safety. They will be asked to evaluate the trial with the following stopping rules: (1) The treatment (defined using the primary outcome from the main trial of repeat violent injury) is significantly worse $(p<0.05),(2)$ the treatment is significantly better $(\mathrm{p}<0.05)$, (3) major safety concerns are identified and protocol changes must occur and (4) it is futile to proceed due to poor recruitment or the control event rate is too low to allow for an obvious difference.

\section{Data collection}

Collection of data will occur through a combination of administrative data through the MCHP and chart review for all members of the cohort and through our textmessaging survey approach with those who are enrolled in the wraparound care arm. The MCHP houses the Population Health Research Data Repository, which allows researchers to anonymously link healthcare data with a multitude of social databases such as education, income, housing and justice, and registries such as the vital statistics data. We will have access to information on any repeat visit to any hospital in Winnipeg, Manitoba, and will be able to link the participant's provincial health insurance number to these social databases. The unique data available at MCHP offer the opportunity to lend novel knowledge to this field by using administrative data to measure visits to any ED and avoiding the need to gather self-report data that is plagued with poor follow-up in this difficult to follow population. Following the identification of a visit to the ED in the year post the index injury (ie, the injury at which time the youth participant was enrolled), a chart review at that hospital will be completed. All outcome assessors and data analysts will be blinded to the treatment arm. The textmessaging survey allows us to determine adherence to the programme (through the WAI survey).

\section{Ethics and dissemination}

The ethics board reviews all amendments, and updates are made on clinicaltrials.gov as required. Informed consents and assents are appended. Study sponsors have no role in the design, collection, management, analysis, interpretation or dissemination of this study.

Dissemination of the study results will occur after review with the Data Safety and Monitoring Board. Initially, study results will be reviewed with the advisory board, which includes clinicians, researchers, community youth workers with expertise in wraparound care, Aboriginal elders and former gang members. Many of the advisory board members were involved in the development of the intervention. All participants will be provided with results. Dissemination of results of the pilot will occur by way of conference presentations and journal articles. No participant level information will be made available.

\section{STUDY STATUS}

Operating grants received from the Canadian Institute of Health Research and the Manitoba Institute of Child Health fund this study. The pilot project was launched in November 2013 and is ongoing.

\section{DISCUSSION}

Breaking the cycle of violence for youth requires a complex, long-term solution. The development of the intervention using a community-based participatory approach demonstrates the importance of involving stakeholders early to ensure that the intervention and the research design are relevant as well as feasible within the community.

Taking advantage of the teachable moment in the ED is theorised to be an opportunity for starting this process with youth. Using a support worker who can develop a relationship quickly in order to take advantage of this teachable moment, and then providing wraparound care immediately and out in the community, are felt to be key to helping youth address the issues that will place them at high risk of a recurrent intentional injury.

The protocol presented in this manuscript demonstrates the importance of completing a pilot of a unique RCT design that will ensure a rigorous evaluation of a complex intervention. This unique randomisation protocol will avoid the potential emotional trauma associated with randomisation to a control group, and protect the participants' right to autonomy over their information and involvement in research. Finally, the development of a cohort of individuals who are injured by violence who can be randomised from within allows a researcher to develop and evaluate various interventions specific to the subgroup that is affected. For example, this cohort will allow us to understand which remote communities have a high incidence of violence and to develop partnerships for future intervention studies. Additionally, in the future, this team plans to develop specific interventions for youth who visit the ED with injuries due to selfharm. Using information gleaned from this cohort, we will be able to better develop appropriate interventions.

\footnotetext{
Author affiliations

${ }^{1}$ Faculty of Health Sciences, Department of Emergency Medicine, University of Manitoba, Winnipeg, Manitoba, Canada

${ }^{2}$ Children's Hospital Research Institute of Manitoba, University of Manitoba, Winnipeg, Manitoba, Canada

${ }^{3}$ Faculty of Health Sciences, Department of Community Health Sciences, University of Manitoba, Winnipeg, Manitoba, Canada

${ }^{4}$ Faculty of Health Sciences, Department of Surgery, University of Manitoba, Winnipeg, Manitoba, Canada
}

Acknowledgements The authors would like to thank the integrated knowledge translation team that helped to design the intervention and 
research approach for this study. They include: Heather Woodward, Nathan Thomas, Elaine Mordoch, Wanda Chernomas, Jamil Mahmood, Floyd Wiebe, Karen Cook, Terry Hammerback, Margaret Lavallee, Justin Rivera, Rob Robinson, Linda Rost, Leslie Spillett and Liz Wolff. They also thank Heather Tiede for her editing expertise.

Contributors CS, DJ, TS, SL and TK were involved in the development of this protocol. CS, DJ, SL and TK were instrumental in development of the adapted research design. DJ is responsible for the analytic plan. TS is responsible for designing the unique automatic notification associated with the screening and recruitment plan. All authors have critically reviewed the manuscript.

Funding Canadian Institute of Health Research (reference number 130476) and Manitoba Institute of Child Health.

Competing interests None declared.

Ethics approval University of Manitoba Health Research Ethics Board Approvals: HS 16444 (2013:257) and HS 16445 (H2013:258).

Provenance and peer review Not commissioned; peer reviewed for ethical and funding approval prior to submission.

Open Access This is an Open Access article distributed in accordance with the Creative Commons Attribution Non Commercial (CC BY-NC 4.0) license, which permits others to distribute, remix, adapt, build upon this work noncommercially, and license their derivative works on different terms, provided the original work is properly cited and the use is non-commercial. See: http:// creativecommons.org/licenses/by-nc/4.0/

\section{REFERENCES}

1. Macpherson A, Schull M, Manuel D, et al. Injuries in Ontario. ICES Atlas. Toronto: Institute for Clinical Evaluative Sciences, 2005.

2. Public Health Agency of Canada. Facts on injury. Secondary facts on injury. 2006. http://www.phac-aspc.gc.ca/injury-bles/facts-eng.php
3. Canadian Institute for Health Information. Intentional assault among children and youth in Canada. Ottawa, ON, 2014.

4. Perreault S. Homicide in Canada, 2011. Juristat 2012(85-002-X)

5. Statistics Canada. CANSIM Table 253-0003-Homicide survey, victims and persons accused of homicide, by age group and sex Canada, annual. 2014.

6. Sims DW, Bivins BA, Obeid FN, et al. Urban trauma: a chronic recurrent disease. J Trauma 1989;29:940-6.

7. Recurrent intentional injury among youth: a Chronic Condition Canadian Injury Prevention Conference. Montreal: 2013.

8. MacPherson A, Jones-Keeshig D, Pike I. Injury rates in Canadian Ontario first nation communities. Inj Prev 2010;16(Suppl 1):A256.

9. Johnson S, Bradshaw C, Wright J, et al. Characterizing the teachable moment: is an emergency department visit a teachable moment for intervention among assault-injured youth and their parents? Pediatr Emerg Care 2007;23:553-9.

10. The National Wraparound Initiative. Secondary The National Wraparound Initiative. http://www.nwi.pdx.edu/index.shtm

11. Skogan W, Harnett SM, Bump N, et al. Executive summary evaluation of CeaseFire-Chicago. 2008.

12. Snider CE, Kirst M, Abubakar S, et al. Community-based participatory research: development of an emergency department-based youth violence intervention using concept mapping. Acad Emerg Med 2010;17:877-85.

13. Campbell R, Peters T, Grant C, et al. Adapting the randomized consent (Zelen) design for trials of behavioural interventions for chronic disease: feasibility study. J Health Serv Res Policy 2005;10:220-5.

14. Relton C, Torgerson D, O'Cathain A, et al. Rethinking pragmatic randomised controlled trials: introducing the "cohort multiple randomised controlled trial" design. BMJ 2010;340:1066.

15. Altman DG, Whitehead J, Parmar MK, et al. Randomised consent designs in cancer clinical trials. Eur J Cancer 1995;31:1934-44.

16. Treatment fidelity and quality control. National Institute of Health Summer Institute on randomized behavioral clinical trials; $18 \mathrm{Jul}$ 2012, 2012; Airlie, VA.

17. Horvath A, Greenberg L. Development and validation of the Working Alliance Inventory. J Couns Psychol 1989;36:223-33. 